\title{
A model for predicting nosocomial carbapenem-resistant Klebsiella pneumoniae infection
}

\author{
DUO YANG $^{1}$, ZEQIANG XIE ${ }^{2}$, XULI XIN $^{2}$, WENYING XUE $^{3}$ and MAN ZHANG $^{2}$ \\ ${ }^{1}$ Central Laboratory; ${ }^{2}$ Clinical Laboratory; ${ }^{3}$ Hospital Infection Management Office, \\ Beijing Shijitan Hospital of Capital Medical University, Beijing 100038, P.R. China
}

Received March 9, 2016; Accepted July 11, 2016

DOI: $10.3892 /$ br.2016.752

\begin{abstract}
Mortality associated with infections due to carbapenem-resistant Klebsiella pneumoniae (CR-KP) is high and the infections need to be predicted early. The risk factors for CR-KP infection are heterogeneous. The aim of the present study was to construct a model allowing for the early prediction of CR-KP infection. Nosocomial infections due to $K$. pneumoniae were evaluated retrospectively over a 2 -year period. The case cohort consisted of 370 inpatients with CR-KP infection. For each case enrolled, two matched controls with no CR-KP infection during their hospitalization were randomly selected. Matching involved month of admission, ward, as well as interval days. The Vitek 2 system was used for identification of isolates and antimicrobial susceptibility testing. General linear model with logistic regression was used to identify possible risk factors. The predicted power of the model was expressed as the area under the receiver-operating characteristic curve. Age, male gender, with cardiovascular disease, hospital stay, recent admission to intensive care unit, indwelling urinary catheter, mechanical ventilation, recent $\beta$-lactam- $\beta$-lactamase inhibitors, fourth-generation cephalosporins and/or carbapenems therapy were independent risk factors for CR-KP infection. Models predicting CR-KP infection developed by cumulative risk factors exhibited good power, with areas under the receiver-operating characteristic curves of 0.902 [95\% confidence interval (CI), 0.883-0.920; $\mathrm{P}<0.001]$ and 0.899 (95\% CI, 0.877-0.921; $\mathrm{P}<0.001)$ after filtering by age ( $\geq 70$ years). The Yonden index was at the
\end{abstract}

Correspondence to: Professor Man Zhang, Clinical Laboratory Beijing Shijitan Hospital of Capital Medical University, 10 Yangfangdian Tieyi Road, Beijing 100038, P.R. China

E-mail: zhang2009man@126.com

Abbreviations: CR-KP, Carbapenem-resistant Klebsiella pneumonia; CS-KP, Carbapenem-sensitive Klebsiella pneumonia; ICU, intensive care unit; ESBLs, extended-spectrum $\beta$-lactamases; MIC, minimal inhibitory concentration; AUC, area under the curve; MDR, multidrug resistance

Key words: nosocomial infection, Klebsiella pneumoniae, carbapenem resistance, risk factors maximum when the cumulative risk factors were $\geq 3$ in the two prediction models. The results show that the prediction model developed in the present study might be useful for controlling infections caused by CR-KP strains.

\section{Introduction}

Infections caused by carbapenem-resistant Klebsiella pneumoniae (CR-KP) isolates are worldwide. The prevalence of $\mathrm{CR}-\mathrm{KP}$ infection in areas of endemicity may vary between 20 and $40 \%$. In addition, these infections often occur in debilitated and immunocompromised patients, in association with prolonged hospital stays (1). The isolates are often resistant to multiple antibiotics, and the mortality associated with infections due to CR-KP is extremely high (2-4). Early identification of possible CR-KP-infected patients and implementation of proper precaution are core measures for controlling CR-KP infections.

Risk factors involved in CR-KP infections have been previously investigated (5-8). These factors were heterogeneous. A retrospective study was conducted in a Chinese tertiary care hospital to identify the main factors associated with nosocomial CR-KP infections, and a model was established for the early prediction of patients with such infection. The results show that the prediction model developed in the present study might be useful for controlling infections caused by CR-KP strains.

\section{Materials and methods}

Setting and participants. The Beijing Shijitan Hospital of the Capital Medical University is a 1,100-bed tertiary care hospital in Beijing, China, with a 26-bed general intensive care unit (ICU), an 8-bed cardiology ICU, an 8-bed respiratory ICU, and a 6-bed emergency medicine ICU.

This retrospective study was conducted based on the hospital electronic database. During the 2-year study period (from January 1, 2012 to December 31, 2013), patients with K. pneumoniae nosocomial infection were evaluated. During the study period, rectal swab screening was not a routine admission procedure and patients were clustered within at least $48 \mathrm{~h}$ once CR-KP infection was confirmed.

In one hospitalization period, each patient was evaluated only once at the time of the index culture (nosocomial 
infections were validated by trained infection management doctors according to the criteria based on a previous study) (9), and the index culture was defined with the $K$. pneumoniae strain first isolated from a clinical specimen and then the corresponding nosocomial infection was confirmed. Patients with CR-KP infections were defined as cases. For each case enrolled, two matched controls with no CR-KP infection during their hospitalization were randomly selected. Matching involved month of admission, ward, as well as interval days (interval from admission to confirmation of the index culture). The length of the entire hospital stay of the controls was equal or more to the interval days of the matched cases.

The following patient data were extracted: Age; gender; transfer from another hospital; comorbidity (at the time of index culture); recent admission to ICU (defined as patients admitted to ICU for $>24 \mathrm{~h}$ before the index culture in one hospitalization period); with CR-KP-positive patients in nearby beds (defined as patients with CR-KP-positive patients in the same ward for $>24 \mathrm{~h}$ before the index culture within the hospitalization period); adopted invasive procedures including central venous catheterization, urinary catheter, nasogastric tube, surgical drain, and invasive mechanical ventilation (at the time of the index culture); and on prior antibiotic therapy (defined as the use of a systemic antimicrobial agent for $\geq 48 \mathrm{~h}$ within the preceding 10 days of index culture in one hospitalization period).

Approval for the study was obtained from the ethics committee of the Beijing Shijitan Hospital of Capital Medical University.

Microbiological procedures. The Vitek 2 system (bioMérieux, Marcy l'Étoile, France) was used for identification of isolates and antimicrobial susceptibility testing. The production of extended-spectrum $\beta$-lactamases (ESBLs) was suggested following use of the Advanced Expert System of the Vitek 2 system. Susceptibility results to carbapenems (imipenem, meropenem, and ertapenem) were interpreted according to Clinical Laboratory Standards Institute guidelines coevally (i.e., susceptibility results of year 2012 were interpreted according to 2012 guideline and results of year 2013 were interpreted according to 2013 standards) (10). Isolates were considered CR-KP if any of the carbapenems (ertapenem, meropenem, and imipenem) was resistant. If the carbapenem MIC of one isolate was intermediary, the isolate was excluded from the analysis.

Statistical analysis. The SPSS version 17.0 (SPSS,Inc.,Chicago, IL, USA) software was used for data analysis. Non-normally distributed continuous variables were reported as medians (interquartile range); and numbers and percentages were reported for categorical variables. The Mann-Whitney U test was used to compare non-normally distributed continuous variables (i.e., ages). Categorical variables were evaluated with the Chi-square test.

The multivariate logistic regression model was used to adjust for potential confounding. Variables associated with CR-KP infection in the univariate analysis $(\mathrm{P} \leq 0.05)$ were included in a logistic regression analysis to identify those independently associated with the development of CR-KP nosocomial infection. Statistical tests were two-tailed. $\mathrm{P} \leq 0.05$ was considered statistically significant.
The power of age and cumulative risk factors to discriminate cases and controls were expressed as the area under the receiver-operating characteristic curve.

\section{Results}

Characteristics of isolates. During the 2-year study period, 370 non-repetitive episodes of nosocomial infections due to CR-KP were found. The characteristics of the cases and controls are shown in Table I. The median (IQR) time of interval days in the case cohort was 17 days (13-21 days). No active outbreak of CR-KP during the 2-year study period was recorded.

CR-KP isolates were recovered from the sputum/bronchoalveolar lavage fluid $(n=268)$, urine $(n=48)$, blood $(n=35)$, skin/mucosa $(n=10)$, surgical site $(n=7)$, and cerebrospinal fluid $(n=2)$. ESBLs were produced by $61 \%$ of CR-KP strains $(n=225)$.

Risk factors associated with CR-KP infection. The main findings of the univariate and multivariate analysis associated with CR-KP infection are shown in Table I. The multivariate analysis revealed that age, male gender, with cardiovascular disease, recent admission to ICU, indwelling urinary catheter and/or surgical drain, mechanical ventilation, and prior administration of $\beta$-lactam- $\beta$-lactamase inhibitors, fourth-generation cephalosporins, and/or carbapenems were significantly different between CR-KP infections and controls.

Cumulative risk factors and their predicting power of CR-KP infection. Male gender, with cardiovascular disease, recent admission to ICU, indwelling urinary catheter, mechanical ventilation, and prior use of antibiotics of $\beta$-lactam- $\beta$-lactamase inhibitors, fourth-generation cephalosporins and/or carbapenems were included in the calculation of cumulative risk factors (Table II).

The area under the curve (AUC) was 0.739 (95\% CI, 0.709-0.768; $\mathrm{P} \leq 0.001)$ for age prediction CR-KP infection. The Yonden index was at its maximum when the patient age was 70 years. After filtering by age ( $\geq 70$ years), 338 CR-KP infection cases and 437 controls remained.

The sensitivity, specificity, and total consistency rate for predicting CR-KP infection at different cut-off levels using cumulative risk factors are shown in Table III. The AUC was 0.902 (95\% CI, 0.883-0.920; P $\leq 0.001)$ for cumulative risk factors predicting $\mathrm{CR}-\mathrm{KP}$ infection tested in the total 1,110 patients and $0.899(95 \% \mathrm{CI}, 0.877-0.921 ; \mathrm{P} \leq 0.001)$ tested in the 775 patients filtered by age (Fig. 1), respectively. The Yonden index was at its maximum when the cumulative risk factors were $\geq 3$ in the two prediction models. However, the sensitivity of prediction increased from 84.8 to $85.2 \%$ and the specificity increased from 68.1 to $81.2 \%$ with age ( $\geq 70$ years), respectively.

\section{Discussion}

The results of the present study showed that age, male gender, with cardiovascular disease, recent admission to ICU for more than $24 \mathrm{~h}$, indwelling urinary catheter, mechanical ventilation, exposure to $\beta$-lactam- $\beta$-lactamase inhibitors, fourth-generation 
Table I. The characteristics of cases and controls.

\begin{tabular}{|c|c|c|c|c|c|c|}
\hline \multirow[b]{2}{*}{ Variables } & \multicolumn{3}{|c|}{ Univariate analysis } & \multicolumn{3}{|c|}{ Multivariate analysis } \\
\hline & Case $(n=370)$ & Control $(n=740)$ & P-value & OR & $95 \% \mathrm{CI}$ & P-value \\
\hline \multicolumn{7}{|l|}{ Demographics } \\
\hline Age (year) & $85(80-87)$ & $74(59-84)$ & $<0.001$ & 1.08 & $1.06-1.10$ & $<0.001$ \\
\hline Male & $321(86.8)$ & $434(58.6)$ & $<0.001$ & 3.839 & $2.36-6.26$ & $<0.001$ \\
\hline Transfer from another hospital & $23(6.2)$ & $16(2.2)$ & 0.001 & 0.750 & $0.29-1.94$ & 0.553 \\
\hline \multicolumn{7}{|l|}{ Comorbidities $^{\mathrm{a}}$} \\
\hline Cardiovascular disease & $80(21.6)$ & $32(15.0)$ & $<0.001$ & 10.00 & $4.52-22.15$ & $<0.001$ \\
\hline Chronic pulmonary disease & $162(43.8)$ & $303(40.9)$ & 0.366 & & & \\
\hline Cerebrovascular disease & $88(23.8)$ & $192(25.9)$ & 0.434 & & & \\
\hline Liver disease & $22(5.9)$ & $50(6.8)$ & 0.605 & & & \\
\hline Chronic renal failure & $27(7.3)$ & $51(6.9)$ & 0.803 & & & \\
\hline Diabetes mellitus & $23(6.2)$ & $43(5.8)$ & 0.788 & & & \\
\hline Peptic ulcer disease & $8(3.0)$ & $32(4.3)$ & 0.068 & & & \\
\hline Malignancy & $26(7.0)$ & $56(7.6)$ & 0.746 & & & \\
\hline Recent surgery ${ }^{b}$ & $36(9.7)$ & $101(13.6)$ & 0.061 & & & \\
\hline \multicolumn{7}{|l|}{ Hospitalization data } \\
\hline Admission to $\mathrm{ICU}^{\mathrm{c}}$ & $225(60.8)$ & $75(10.1)$ & $<0.001$ & 2.06 & $1.03-4.10$ & 0.040 \\
\hline CR-KP-positive patients in nearby beds $\mathrm{s}^{\mathrm{d}}$ & $251(67.8)$ & $111(15.0)$ & $<0.001$ & 1.18 & $0.60-2.29$ & 0.636 \\
\hline \multicolumn{7}{|l|}{ Invasive procedures $^{a}$} \\
\hline Central venous catheter & $198(53.5)$ & $109(14.7)$ & $<0.001$ & 1.64 & $0.99-2.71$ & 0.052 \\
\hline Urinary catheter & $297(80.2)$ & $240(32.4)$ & $<0.001$ & 2.21 & $1.36-3.59$ & 0.001 \\
\hline Nasogastric tube & $207(55.9)$ & $65(8.8)$ & $<0.001$ & 0.95 & $0.41-2.23$ & 0.904 \\
\hline Surgical drain & $10(2.7)$ & $22(3.0)$ & 0.800 & & & \\
\hline Mechanical ventilation & $193(52.2)$ & $56(7.6)$ & $<0.001$ & 2.80 & $1.15-6.86$ & 0.024 \\
\hline \multicolumn{7}{|l|}{ Prior antibiotic therapy ${ }^{\mathrm{e}}$} \\
\hline Second-generation cephalosporins & $40(10.8)$ & $335(45.2)$ & $<0.001$ & 0.787 & $0.43-1.43$ & 0.433 \\
\hline Third-generation cephalosporins & $65(17.6)$ & $102(13.8)$ & 0.096 & & & \\
\hline Fourth-generation cephalosporins & $27(7.3)$ & $18(2.4)$ & $<0.001$ & 3.79 & $1.44-10.03$ & 0.007 \\
\hline$\beta$-lactam- $\beta$-lactamase inhibitors & $156(42.2)$ & $111(15.0)$ & $<0.001$ & 10.65 & $5.93-19.10$ & $<0.001$ \\
\hline Carbapenems & $194(52.4)$ & $123(16.6)$ & $<0.001$ & 7.75 & $4.21-14.27$ & $<0.001$ \\
\hline Fluoroquinolones & $47(12.7)$ & $106(14.3)$ & 0.460 & & & \\
\hline Aminoglycosides & $25(6.8)$ & $38(5.1)$ & 0.271 & & & \\
\hline Others $^{\mathrm{f}}$ & $55(14.9)$ & $137(18.5)$ & 0.130 & & & \\
\hline
\end{tabular}

Results are shown as number (\%) or median (range). CR-KP, carbapenem-resistant Klebsiella pneumoniae; OR, odds ratio; CI, confidence interval; ICU, intensive care unit. ${ }^{\mathrm{a} A t}$ the time of the index culture. ${ }^{\mathrm{b}}$ Surgical procedure performed within 14 days before the index culture. ${ }^{\mathrm{c}} \mathrm{Admitted} \mathrm{to} \mathrm{ICU}$ for more than $24 \mathrm{~h}$ before the index culture within the hospitalization period. ${ }^{\mathrm{d}} \mathrm{A} \mathrm{CR}-\mathrm{KP}$-positive patient in the nearby bed for more than $24 \mathrm{~h}$ before the index culture within the hospitalization period. ${ }^{\text {ZZero to }} 30$ days before the index culture within the hospitalization period. Including semisynthetic penicillins, oxyimino-cephalosporins, glycopeptides, linezolid, rifampin, erythromycins, and antifungal drugs.

cephalosporins and carbapenems were associated with CR-KP infection.

Compared with young patients, elderly patients were more likely to have comorbidities and susceptibility to infections (11). In the present study, patients with CR-KP infection were elderly compared to those with no CR-KP infection.

Male gender was associated with CR-KP infection. Daikos et al found that the proportion of male patients surviving was significantly higher than those that succumbed due to carbapenemase-producing $K$. pneumoniae bloodstream infections (2). However, to the best of our knowledge, this is the first time that the male gender and cardiovascular disease constituted risk factors for CR-KP infection. Regarding the latter, it may be explained by the fact that cardiovascular disease is diagnosed more frequently in men (12).

As expected, ICU stay was an independent risk factor for CR-KP infections. ICUs are the principal hospital reservoirs of MDR bacteria. Patients with ICU admission represent a population subjected to many invasive procedures and medical devices, which facilitate cross-transmission of resistance mechanisms $(7,13,14)$. 
Table II. Distribution of cumulative risk factors for CR-KP infection.

\begin{tabular}{lrrrrrr}
\hline No. of risk factors & Case $^{\mathrm{a}}$ & Control $^{\mathrm{a}}$ & Total $^{\mathrm{a}}$ & Case $^{\mathrm{b}}$ & Control $^{\mathrm{b}}$ & Total $^{\mathrm{b}}$ \\
\hline 0 & 4 & 154 & 158 & 4 & 93 & 97 \\
1 & 13 & 279 & 292 & 11 & 160 & 171 \\
2 & 39 & 171 & 210 & 35 & 102 & 137 \\
3 & 76 & 94 & 170 & 71 & 54 & 125 \\
4 & 86 & 29 & 115 & 86 & 19 & 105 \\
5 & 86 & 13 & 99 & 71 & 9 & 80 \\
6 & 66 & 0 & 66 & 60 & 0 & 60 \\
7 & 0 & 0 & 0 & 0 & 0 & 0 \\
Total & 370 & 740 & 1,110 & 338 & 437 & 775 \\
\hline
\end{tabular}

Risk factors included the following: Male gender, cardiovascular disease, recent admission to intensive care unit, urinary catheter, mechanical ventilation, fourth-generation cephalosporins therapy, $\beta$-lactam- $\beta$-lactamase inhibitors therapy, and carbapenems therapy. ${ }^{\text {a }}$ Results are shown as numbers and tested in total 1,110 (370 cases and 740 matched controls) patients. ${ }^{b}$ Results are shown as numbers and tested in 775 patients [338 CR-KP infection positive cases and 437 matched controls after filtering by age ( $\geq 70$ years)]. CR-KP, carbapenem-resistant Klebsiella pneumoniae.

Table III. Sensitivity, specificity, and total consistency rate for predicting CR-KP infection at different cut-off levels.

\begin{tabular}{lccccrr}
\hline No. of risk factors & $\mathrm{SE}^{\mathrm{a}}$ & $\mathrm{SP}^{\mathrm{a}}$ & $\mathrm{TCR}^{\mathrm{a}}$ & $\mathrm{SE}^{\mathrm{b}}$ & $\mathrm{SP}^{\mathrm{b}}$ & $\mathrm{TCR}^{\mathrm{b}}$ \\
\hline$\geq 1$ & 98.9 & 20.8 & 46.8 & 98.8 & 21.3 & 55.1 \\
$\geq 2$ & 95.4 & 58.5 & 70.8 & 95.6 & 57.9 & 774.3 \\
$\geq 3$ & 84.8 & 68.1 & 82.7 & 85.2 & 81.2 & 83.0 \\
$\geq 4$ & 64.3 & 94.3 & 84.3 & 64.2 & 93.6 & 80.7 \\
$\geq 5$ & 41.1 & 98.2 & 79.2 & 38.8 & 97.9 & 72.1 \\
$\geq 6$ & 17.8 & 100.0 & 72.6 & 17.8 & 100.0 & 64.1 \\
\hline
\end{tabular}

Risk factors included the following: Male gender, cardiovascular disease, recent admission to intensive care unit, urinary catheter, mechanical ventilation, fourth-generation cephalosporins therapy, $\beta$-lactam- $\beta$-lactamase inhibitors therapy, and carbapenems therapy. SE, sensitivity; SP, specificity; TCR, total consistency rate. ${ }^{a}$ Results are shown as percentage and tested in total 1,110 (370 cases and 740 matched controls) patients. ${ }^{b}$ Results are shown as percentage and tested in 775 patients [338 CR-KP infection positive cases and 437 matched controls after filtered by age ( $\geq 70$ years)]. CR-KP, carbapenem-resistant Klebsiella pneumoniae.

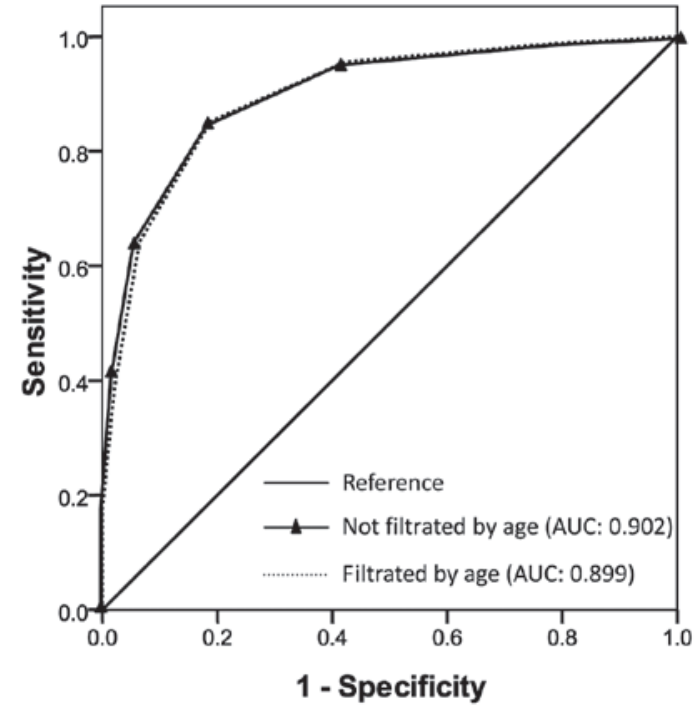

Figure 1. Receiver-operating characteristic curves for cumulative risk factors predicting carbapenem-resistant Klebsiella pneumoniae infection. AUC, area under the curve.
In the current study, CR-KP infection was also associated with indwelling urinary catheter and mechanical ventilation. These invasive procedures are well-known risk factors for MDR bacteria infection/colonization $(7,13,15)$.

Prior administration of $\beta$-lactam- $\beta$-lactamase inhibitors and/or carbapenems was associated with CR-KP infection, as reported in other studies $(7,8,16)$. This observation may be due to the fact that treatment with broad-spectrum antibiotics potentially promotes the selection of CR-KP strains by eliminating susceptible competing clones. In a previous study, it was reported that a combination therapy containing carbapenem was strongly associated with survival in CR-KP bloodstream infections (2). Thus, administration of carbapenem should be used prudently in empirical therapy, and once the CR-KP infection is highly suspected or verified, a regimen including carbapenem is likely to benefit the outcome.

Previous findings have shown that the presence of CR-KP-colonized patients in nearby beds was associated with CR-KP infection/colonization $(17,18)$. In the present study, although the proportion of patients who had CR-KP-positive 
patients in nearby beds was higher in the case group than in the control group, CR-KP-positive patients in nearby beds was not an independent risk factor of CR-KP infection. This discrepancy may partly be because of the different case-control design, and remains to be confirmed.

The model for predicting CR-KP infection developed in this study displayed good discriminatory power (AUC $=0.902)$, which is similar to the model developed by Tumbarello et al (7). The sensibility was $84.8 \%$ and specificity was $68.1 \%$ when there were three or more risk factors for predicting CR-KP infection. The age of patients ( $\geq 70$ years) increased the predicting specificity to $81.2 \%$. The predicting model is potentially useful in hospitals where CR-KP infection is epidemic. Empirical application of infection control measures, such as rectal swabs, may be limited to individuals with a higher possibility of CR-KP infection, thereby reducing workloads and costs. This prediction model may also be useful for empirical antimicrobial therapy and potentially reduce the risk of ineffective therapy during the initial phase of treatment. Notably, the prevention of nosocomial acquisition of resistant bacteria is dependent on implementation of and adherence to infection control standards.

In conclusion, the present findings provide information that may be useful for identifying patients at high risk for CR-KP infection and may assist physicians to use more effective treatments for CR-KP infections.

\section{References}

1. Tzouvelekis LS, Markogiannakis A, Psichogiou M, Tassios PT and Daikos GL: Carbapenemases in Klebsiella pneumoniae and other Enterobacteriaceae: An evolving crisis of global dimensions. Clin Microbiol Rev 25: 682-707, 2012.

2. Daikos GL, Tsaousi S, Tzouvelekis LS, Anyfantis I, Psichogiou M, Argyropoulou A, Stefanou I, Sypsa V, Miriagou V, Nepka M, et al: Carbapenemase-producing Klebsiella pneumoniae bloodstream infections: Lowering mortality by antibiotic combination schemes and the role of carbapenems. Antimicrob Agents Chemother 58: 2322-2328, 2014.

3. Fraenkel-Wandel Y, Raveh-BrawerD, Wiener-Well Y, Yinnon AM and Assous MV: Mortality due to blaKPC Klebsiella peumoniae bacteraemia. J Antimicrob Chemother 71: 1083-1087, 2016.

4. QureshiZA, Paterson DL, Potoski BA, Kilayko MC, Sandovsky G, Sordillo E, Polsky B, Adams-Haduch JM and Doi Y: Treatment outcome of bacteremia due to KPC-producing Klebsiella pneumoniae: Superiority of combination antimicrobial regimens. Antimicrob Agents Chemother 56: 2108-2113, 2012.

5. Falagas ME, Rafailidis PI, Kofteridis D, Virtzili S, Chelvatzoglou FC, Papaioannou V, Maraki S, Samonis G and Michalopoulos A: Risk factors of carbapenem-resistant Klebsiella pneumoniae infections: A matched case control study. J Antimicrob Chemother 60: 1124-1130, 2007.
6. Schwaber MJ, Klarfeld-Lidji S, Navon-Venezia S, Schwartz D, Leavitt A and Carmeli Y: Predictors of carbapenem-resistant Klebsiella pneumoniae acquisition among hospitalized adults and effect of acquisition on mortality. Antimicrob Agents Chemother 52: 1028-1033, 2008.

7. Tumbarello M, Trecarichi EM, Tumietto F, Del Bono V, De Rosa FG, Bassetti M, Losito AR, Tedeschi S, Saffioti C, Corcione S, et al: Predictive models for identification of hospitalized patients harboring KPC-producing Klebsiella pneumoniae. Antimicrob Agents Chemother 58: 3514-3520, 2014.

8. Zarkotou O, Pournaras S, Voulgari E, Chrysos G, Prekates A, Voutsinas D, Themeli-Digalaki K and Tsakris A: Risk factors and outcomes associated with acquisition of colistin-resistant KPC-producing Klebsiella pneumoniae: A matched case-control study. J Clin Microbiol 48: 2271-2274, 2010.

9. Horan TC, Andrus M and Dudeck MA: CDC/NHSN surveillance definition of health care-associated infection and criteria for specific types of infections in the acute care setting. Am J Infect Control 36: 309-332, 2008.

10. Clinical and Laboratory Standards Institute (CLSI): Methods for dilution antimicrobial susceptibility tests for bacteria that grow aerobically; Approved standard M100. CLSI, Wayne, PA, 2012.

11. Rodríguez-Baño J, Alcalá JC, Cisneros JM, Grill F, Oliver A, Horcajada JP, Tórtola T, Mirelis B, Navarro G, Cuenca M, et al: Community infections caused by extended-spectrum beta-lactamase-producing Escherichia coli. Arch Intern Med 168: 1897-1902, 2008.

12. Pilote L, Dasgupta K, Guru V, Humphries KH, McGrath J, Norris C, Rabi D, Tremblay J, Alamian A, Barnett T, et al: A comprehensive view of sex-specific issues related to cardiovascular disease. CMAJ 176: S1-S44, 2007.

13. Trecarichi EM, Cauda R and Tumbarello M: Detecting risk and predicting patient mortality in patients with extended-spectrum $\beta$-lactamase-producing Enterobacteriaceae bloodstream infections. Future Microbiol 7: 1173-1189, 2012.

14. Lucena A, Dalla Costa LM, Nogueira KS, Matos AP, Gales AC, Paganini MC, Castro ME and Raboni SM: Nosocomial infections with metallo-beta-lactamase-producing Pseudomonas aeruginosa: Molecular epidemiology, risk factors, clinical features and outcomes. J Hosp Infect 87: 234-240, 2014.

15. Lin MY, Lyles-Banks RD, Lolans K, Hines DW, Spear JB, Petrak R, Trick WE, Weinstein RA and Hayden MK; Centers for Disease Control and Prevention Epicenters Program: The importance of long-term acute care hospitals in the regional epidemiology of Klebsiella pneumoniae carbapenemase-producing Enterobacteriaceae. Clin Infect Dis 57: 1246-1252, 2013.

16. Papadimitriou-Olivgeris $M$, Marangos M, Fligou $F$, Christofidou M, Bartzavali C, Anastassiou ED and Filos KS: Risk factors for KPC-producing Klebsiella pneumoniae enteric colonization upon ICU admission. J Antimicrob Chemother 67: 2976-2981, 2012

17. Papadimitriou-Olivgeris M, Christofidou M, Fligou F, Bartzavali C, Vrettos T, Filos KS, Marangos $M$ and Anastassiou ED: The role of colonization pressure in the dissemination of colistin or tigecycline resistant KPC-producing Klebsiella pneumoniae in critically ill patients. Infection 42: 883-890, 2014

18. Bonten MJ: Colonization pressure: A critical parameter in the epidemiology of antibiotic-resistant bacteria. Crit Care 16: 142, 2012. 